\title{
Determination of the Uranium Backgrounds in Lexan Films for Single Particle Analysis using FT-TIMS technique
}

\author{
Sujin Park, Jong-Ho Park, Myung Ho Lee, and Kyuseok Song* \\ Nuclear Chemistry Research Division, Korea Atomic Energy Research Institute, Daejeon 305-353, Korea
}

Received June 1, 2011; Revised June 7, 2011; Accepted June 8, 2011

First published on the web June 15, 2011; DOI: 10.5478/MSL.2011.2.2.057

\begin{abstract}
As background significantly affects measurement accuracy and a detection limit in determination of the trace amounts of uranium, it is necessary to determine the impurities in the Lexan detector film for single particle measurements by thermal ionization mass spectrometry coupled with fission track technique (FT-TIMS). We have prepared various micro sizes of the blank Lexan detector film using a micromanipulation technique for uranium measurements by TIMS. Few tens of fg of uranium background with no remarkable dependency on the film sizes were observed in the blank Lexan films with the sizes from $50 \times 50 \mu \mathrm{m}^{2}$ to $300 \times 300 \mu \mathrm{m}^{2}$. Based on the determination of the uranium background in the Lexan film, any background correction is necessary in the isotopic analysis of a uranium single particle with micron sizes when the particle bearing Lexan film is dissected with less than $300 \times 300 \mu \mathrm{m}^{2}$ size. The isotopic analysis of a uranium particle in U030 standard material using TIMS was carried out to verify the applicability of the Lexan film to the single particle analysis with high accuracy and precision.
\end{abstract}

Key words: Uranium, Background, TIMS, Particle Analysis, Isotopic Analysis, Safeguards

\section{Introduction}

A swipe sample collected from a nuclear facility by an IAEA inspector contains huge numbers of particles together with extremely small amounts of uranium particles originated from nuclear activities. ${ }^{1}$ The amounts of uranium are usually in a few nano-gram levels corresponding to a few thousands uranium particles with micron sizes. The isotopic analysis of uranium single particles contained in a swipe sample plays crucial role in providing direct evidence to estimate the nuclear history of a facility. ${ }^{1}$ For examples, the isotopic ratio of ${ }^{235} \mathrm{U}$ and ${ }^{238} \mathrm{U}$ determines the enrichment of uranium, while the ratios of minor isotopes compared to ${ }^{238} \mathrm{U}$, which are ${ }^{234} \mathrm{U}{ }^{238} \mathrm{U}$ and ${ }^{236} \mathrm{U}{ }^{238} \mathrm{U}$, enable us to estimate the process types.

The single particle analysis requires mainly four procedures; collection, screening, manipulation, and isotopic ratio measurements. The particle collection can be performed by a suction system to transfer the particles from a swipe to a certain substrate, such as a membrane or a graphite planchet, for the next steps. ${ }^{2,3}$ The screening procedure to identify the position of the uranium particles is necessary as most particles collected to a substrate are not uranium bearing particles but just regular dusts consisting mainly of carbon. Although several techniques including X-ray analysis and alpha track method are used for screening, fission track technique which utilizes thermal neutron irradiation from a nuclear reactor is

*Reprint requests to Dr. Kyuseok Song

E-mail: sks@kaeri.re.kr the most promising one from a sensitivity point of view. The particles collected on a substrate are dispersed onto a Lexan detector with a diluted collodion solution followed by being treated with thermal neutron irradiation, which induces fission of uranium to physically scratch the Lexan detector. ${ }^{2,7}$ The scratch can be visible with etching to make tracks. A micromanipulation system is utilized to dissect the particle bearing film and to transfer it onto a measurement device for isotopic ratio determination. Thermal ionization mass spectrometry (TIMS) is one of the most powerful methods for isotopic measurements due to its high accuracy, precision, and sensitivity. ${ }^{2,8-12}$

In most cases, a small background is not considered as a significant correction factor to improve measurement accuracy. As the amount of target materials becomes small, however, the importance of background gradually increases, resulting in a serious distortion in isotope ratio. In the TIMS measurement, most backgrounds come from the impurities in filaments used for sample loading, evaporation, and ionization. Although zone-refined rhenium filaments contain less impurity than normal rhenium filaments, a degassing treatment with high temperature under vacuum conditions is necessary to reduce the background to a negligible level. ${ }^{13}$ In the case of FT-TIMS, additional uranium backgrounds can be originated from the Lexan detector used for particle screening. Therefore, it is necessary to determine the uranium background from the Lexan detector for correction purpose.

In this study, we have prepared blank Lexan detector films with various sizes followed by measuring the uranium backgrounds from them using TIMS. Micromanipulation to 
dissect and transfer the detector films is described. An isotopic analysis of a single particle of a uranium standard reference material has been performed using FT-TIMS technique to verify the applicability of the Lexan detector.

\section{Experimental}

A micromanipulation system equipped with a digital microscope (VHX-Z100R, Kyence) was utilized to prepare the blank Lexan detector films with various sizes. Figure 1 shows the optical image of the dissected Lexan detector films with $50 \times 50 \mu \mathrm{m}^{2}, 100 \times 100 \mu \mathrm{m}^{2}$, and $300 \times 300 \mu \mathrm{m}^{2}$. Three dissected films for each size were prepared. A pair of microprobe with $5 \mu \mathrm{m}$ of end-tip size was used to pick up and transfer the dissected films to a zone-refined rhenium filament. The filaments were pre-degassed under high temperature $\left(1500^{\circ} \mathrm{C}\right)$ and high vacuum conditions to reduce the uranium backgrounds into negligible levels, which was approximately $1 \mathrm{fg}$. The blank Lexan films were fixed on the filaments applying heating current (1.8 A) for $45 \mathrm{sec}$.

The uranium measurement was performed with TIMS (TRITON, Thermo Scientific). A differential pumping system consisting of a turbo-molecular pump and two ion-getter pumps maintained the ultra-high vacuum pressure of the system. In the thermal ionization source electrically floated by $+10 \mathrm{kV}$, a double-filament system evaporated, and then, immediately ionized the sample by considerably high temperature $\left(\sim 1800^{\circ} \mathrm{C}\right)$ followed by pushing the ions with accelerating voltage to a analyzer. A magnetic sector analyzed the ions spatially by masses, which were then transduced into electrical current by a combination of faraday cups and a secondary electron multiplier (SEM).

\section{Results and Discussion}

Determination of uranium background in blank Lexan films

The uranium backgrounds from the blank Lexan films

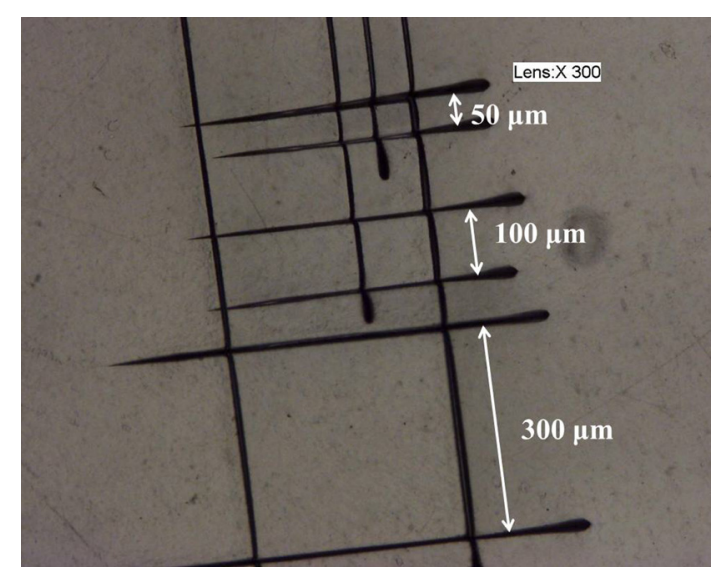

Figure 1 An optical image of the dissected Lexan detector film with $50 \times 50 \mu \mathrm{m}^{2}, 100 \times 100 \mu \mathrm{m}^{2}$, and $300 \times 300 \mu \mathrm{m}^{2}$ size.
Table 1 Uranium backgrounds from the blank Lexan films.

\begin{tabular}{cccc}
\hline \hline & $50 \times 50 \mu \mathrm{m}^{2}$ & $100 \times 100 \mu \mathrm{m}^{2}$ & $300 \times 300 \mu \mathrm{m}^{2}$ \\
\hline${ }^{238} \mathrm{U}$ intensity $(\mathrm{cps}) *$ & $213[ \pm 240]$ & $130[ \pm 122]$ & $307[ \pm 354]$ \\
$\mathrm{U}$ amount (fg) & 40 & 25 & 60 \\
\hline
\end{tabular}

*Numbers in parentheses indicate expanded uncertainties $U=k \cdot u_{c}$

with three different dissected sizes were measured using the SEM as a transducer. As summarized in Table 1, the signal intensities of ${ }^{238} \mathrm{U}$ averaged out three measurements for the sizes of $50 \times 50 \mu \mathrm{m}^{2}, 100 \times 100 \mu \mathrm{m}^{2}$, and $300 \times 300 \mu \mathrm{m}^{2}$ were 213,130 , and $307 \mathrm{cps}$, respectively. The contribution of the uranium backgrounds from the blank filaments was neglected based on the preliminary quantification of the backgrounds. The uncertainties were approximately $100 \%$ due to the ultra-trace level of intensities. The preliminary quantification using isotope dilution mass spectrometry (IDMS) technique, ${ }^{14,15}$ showing that one cps corresponds to approximately $0.2 \mathrm{fg}$ and the limit of quantification (LOQ) is $4.3 \mathrm{fg}$, enables us to estimate the amounts of uranium backgrounds in each dissected sizes to be 40,25 , and $60 \mathrm{fg}$, respectively. Although the numbers possessed no significant meaning due to their extremely big uncertainties closed to $100 \%$ and hence no correlation between the films sizes and the uranium background levels were found, the result showed the uranium background in the blank Lexan film with less than $300 \times 300 \mu \mathrm{m}^{2}$ size is considered to be negligible in the measurements for uranium particles with over pg level. This means that no background correction is necessary for isotopic analysis of single uranium particle with micron sizes since a single uranium oxide particle in $1 \mu \mathrm{m}$ diameter contains approximately $4 \mathrm{pg}$ of uranium and the typical dissection size of a Lexan film for a single particle analysis is $100 \times 100 \mu \mathrm{m}^{2}$.

Although the uranium background in a blank Lexan film up to $300 \times 300 \mu \mathrm{m}^{2}$ size is determined as a negligible quantity, there is necessity to minimize the dissection size of the film. This is because an additional uranium background or interference from co-inclusion of dust particles or a neighboring uranium particle with a target particle in a dissected film is possible. Hence, the whole particles are required to be widely spread out during dispersion process, and special care must be paid in dissection process not to include more than two particles in a dissected film.

Isotope Ratio Measurement of a U030 single particle

In order to verify the applicability of the blank Lexan detector film to the uranium single particle analysis, the isotope ratios of a uranium oxide particle of a standard reference material (NIST U030) were determined using FT-TIMS. Various sizes of U030 particles were dispersed and fixed onto a Lexan detector with a diluted collodion solution. The particle screening procedure with neutron irradiation to induce fission tracks was omitted under the assumption that other than uranium particles were unable to be present on the detector. As shown 

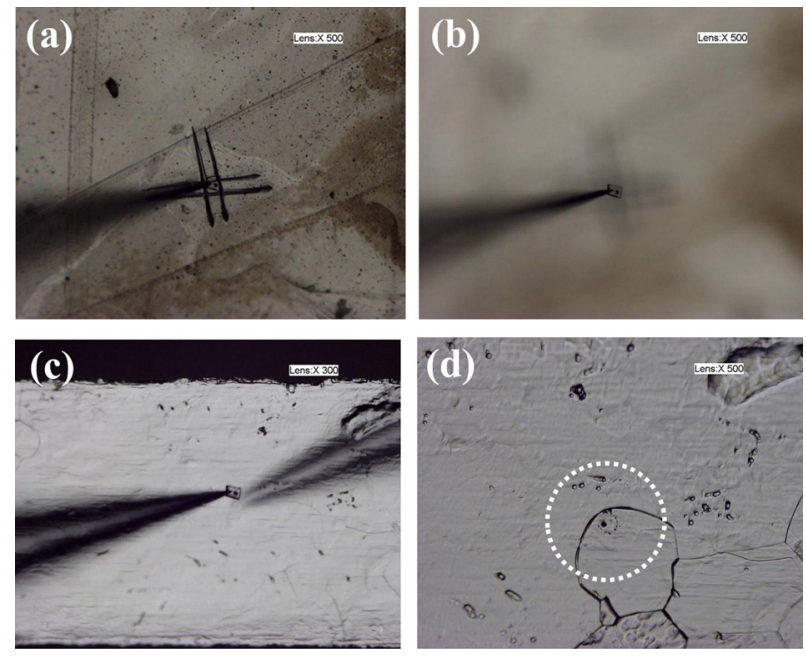

Figure 2. The optical images of particle manipulation; (a), (b) particle bearing Lexan film pickup, (c) particle bearing Lexan film loading on a rhenium filament, (d) the fixed particle on a rhenium filament.

in Figure 2(a), particle pickup of the dissected Lexan film bearing a U030 particle with approximately $5 \mu \mathrm{m}$ size was carried out for particle loading on a rhenium filament (Figure 2(b) and Figure 2(c)). The particle was fixed on the filament by applying heating current with a drop of $1 \mathrm{M}$ nitric acid as shown in Figure 2(d). The total amount of the U030 particle loaded on the filament was approximately $500 \mathrm{pg}$.

The continuous heating method, ${ }^{16}$ in which data collection was performed with gradual increase in the current of evaporation filament (EF) up to $4,500 \mathrm{~mA}$, was applied. The data acquisition method consisted of three steps as shown in Table 2. In each step, the SEM, and a corresponding faraday cup were used for the simultaneous detection of uranium isotopes, which cancels out the signal fluctuation during data acquisition resulting

Table 2. Detector configurations for data acquisition.

\begin{tabular}{|c|c|c|c|c|}
\hline Step & SEM FAR-H1 & FAR-H2 & FAR-H3 & Integration time (s) \\
\hline 1 & ${ }^{234} \mathrm{U}$ & & ${ }^{238} \mathrm{U}$ & 4 \\
\hline 2 & ${ }^{235} \mathrm{U}$ & ${ }^{238} \mathrm{U}$ & & 2 \\
\hline 3 & ${ }^{236} \mathrm{U}$ & & & 4 \\
\hline
\end{tabular}

Table 3. Uranium isotope ratios of an U030 particle with approximately $5 \mu \mathrm{m}$ size.

\begin{tabular}{cccc}
\hline \hline & ${ }^{234} \mathrm{U} /{ }^{238} \mathrm{U}$ & ${ }^{235} \mathrm{U}{ }^{238} \mathrm{U}$ & ${ }^{236} \mathrm{U} /{ }^{238} \mathrm{U}$ \\
\hline Certified Value & $1.960[ \pm 0.010]$ & $3.143[ \pm 0.05]$ & $2.104[ \pm 0.010]$ \\
& $\times 10^{-4}$ & $\times 10^{-2}$ & $\times 10^{-4}$ \\
& $1.945[ \pm 0.021]$ & $3.138[ \pm 0.010]$ & $2.092[ \pm 0.021]$ \\
Measured Value & $\times 10^{-4}$ & $\times 10^{-2}$ & $\times 10^{-4}$ \\
Accuracy (\%) & 0.77 & 0.16 & 0.57 \\
\hline
\end{tabular}

*Accuracy is defined as (measured value - certified value)/(certified value). in enhancing precision. The integration times for the steps were $4 \mathrm{~s}$ and $2 \mathrm{~s}$ for the minor isotopes and ${ }^{235} \mathrm{U}$, respectively.

Table 3 shows the excellent agreement of the measurements with the certified isotopic ratios. The accuracies of the measurement were $0.77 \%, 0.16 \%$, and $0.57 \%$, for ${ }^{234} \mathrm{U} /{ }^{238} \mathrm{U}$, ${ }^{235} \mathrm{U}{ }^{238} \mathrm{U}$, and ${ }^{236} \mathrm{U}{ }^{238} \mathrm{U}$, respectively, with high precision. This implies that the quality control to prevent contamination of the sample was well maintained, and the uranium background in the Lexan film was in a negligible level compared to the uranium amount in the target particle with approximately $5 \mu \mathrm{m}$ size.

As mentioned in the previous section, the interference of the uranium background in Lexan films on the isotopic analysis of single uranium particle with $1 \mu \mathrm{m}$ size is considered to be negligible. Extremely advanced particle manipulation technique and an ultra-sensitive isotope measurement are required to verify the applicability of the Lexan detector film for the analysis of a single particle with $1 \mu \mathrm{m}$ size..$^{16,17}$

\section{Summary}

The uranium backgrounds in the Lexan detector films with the sizes of $50 \times 50 \mu^{2}, 100 \times 100 \mu^{2}$, and $300 \times 300 \mu \mathrm{m}^{2}$ were determined as 40,25 , and $60 \mathrm{fg}$, respectively, to consider the necessity of any background correction for the accurate isotopic analysis of a uranium single particle. An advanced micromanipulation technique was utilized to dissect and to load the Lexan films for the measurement using TIMS. Only negligible amounts of uranium background in the Lexan film were observed with no dependency on the film size. This implies no background correction is necessary in the isotopic analysis of a uranium single particle with micron sizes when the particle bearing Lexan film is dissected with less than $300 \times 300 \mu \mathrm{m}^{2}$ size. The uranium isotope ratio measurement of a U030 particle using TIMS was carried out to verify the applicability of the Lexan film to the single particle analysis with high accuracy and precision.

\section{Acknowledgements}

This work was supported by the long-term nuclear research and development project of the Ministry of Science and Technology of Korea.

\section{References}

1. Donohue, D. L. J. Alloy Compd. 1998, 271-273, 11.

2. Lee, C. G; Iguchi, K.; Esaka, F.; Magara, M.; Sakurai, S.; Watanabe, K.; Usuda, S. Jpn. J. Appl. Phys. 2006, 45, 294.

3. Esaka, K. T; Esaka, F.; Inagawa, J.; Iguchi, K.; Lee, C. G.; Sakurai, S.; Watanabe, K.; Usuda, S., Jpn, J. Appl. Phys. 2004, 43, L915.

4. Lee, M. H; Park, J.-H.; Song, K.; Radiat. Meas. 2011, 46, 409.

5. Shen, Y.; Zhao, Y.; Guo, S.; Cui, J.; Liu, Y.; Li, J.; Xu, J; Zhang, H., Radiat. Meas. 2008, 43, S299. 
6. Lee, C. G; Iguchi, K.; Inagawa, J.; Suzuki, D.; Esaka, F.; Magara, M.; Sakurai, S.; Watanabe, K.; Usuda, S., J. Radioanal. Nucl. Chem. 2007, 272, 299.

7. Lee, M. H.; Douglas, M.; Clark, S. B., Radiat. Meas. 2005, 40, 37 .

8. Cohen, A. S.; Belshaw. N. S.; O’Nions, R. K. Int. J. Mass Spectrom. Ion Process., 1992, 116, 71.

9. Heumann, K. G; Eisenhut, S.; Gallus, S.; Hebeda, E. H.; Nusko, R.; Vengosh, A.; Walczyk, T. Analyst, 1995, 120, 1291.

10. Rubin, K. H. Chemical Geology, 2001, 175, 723.

11. Richter, S.; Goldberg, S. A. Int. J. Mass Spectrom. 2003, 229, 181.
12. Suzuki, D.; Saito-Kokubu, Y.; Sakurai, S.; Lee, C.-G; Magara, M.; Iguchi, K.; Kimura, T. Int. J. Mass Spectrom. 2010, 294, 23.

13. Jeon, Y. S.; Park, Y. J.; Joe, K. S.; Han, S. H.; Song K. Analytical Science and Technology, 2008, 21, 487.

14. Wolff, J. C.; Dyckmans, B.; Taylor P. D. P.; Bievre, P. D. Int. J. Mass Spectrom. Ion Process., 1996, 156, 67.

15. Heumann, K. G Int. J. Mass Spectrom. Ion Phys., 1982, 45, 87.

16. Suzuki, D.; Kokubu, Y. S.; Sakurai, S.; Lee, C. G; Magara, M.; Iguchi, K.; Kimura, T., Int. J. Mass. Spectrom. 2010, 294, 23.

17. Kraiem, M.; Richter, S.; Kuhn, H.; Aregbe, Y. Anal. Chim. Acta 2011, 688, 1. 